\title{
Fatty acid desaturase 2 promoter mutation is not responsible for $\Delta 6$-desaturase deficiency
}

\author{
Melissa K Gregory ${ }^{\star}$, , Susan E Lester ${ }^{2}$, Rebecca J Cook-Johnson ${ }^{1}$, Robert A Gibson ${ }^{3}$, Susanna M Proudman ${ }^{1}$, \\ Leslie G Cleland ${ }^{1}$ and Michael J James ${ }^{1}$
}

Dietary essential polyunsaturated fatty acids (PUFAs) require fatty acid desaturases (FADS) for conversion to long-chain PUFAs (LCPUFAs), which are critical for many aspects of human health. A $\Delta 6$-desaturase deficiency in a single patient was attributed to an insertion mutation in the FADS2 promoter. Later population studies have shown this thymidine nucleotide (T) insertion to be a common polymorphism ( $r s 3834458$ ). We examined correlations between rs3834458 variants and fatty acid evidence of FADS2 activity in a cohort of rheumatoid arthritis patients selected for low or nil consumption of n-3 LCPUFA as fish or fish oil. The presence of the T allele was associated with higher FADS2 activity, as indicated by higher conversion of plasma n-3 PUFA to LCPUFA. However, the T-insertion/deletion polymorphism did not affect FADS2 promoter activity in luciferase reporter assays in HepG2 or NIH/3T3 cells. Our results indicate that the polymorphism rs3834458 does not appear to directly affect FADS2 promoter activity and is not responsible for a previously reported $\Delta 6$-desaturase deficiency.

European Journal of Human Genetics (2011) 19, 1202-1204; doi:10.1038/ejhg.2011.104; published online 1 June 2011

Keywords: FADS2 promoter; polymorphism; polyunsaturated fatty acids; $\Delta 6$-desaturase

\section{INTRODUCTION}

$\Delta 6$-Desaturase is the rate-limiting enzyme for conversion of the dietary essential 18-carbon n-3 and n-6 polyunsaturated fatty acids (PUFAs) to long-chain PUFAs (LCPUFAs). The n-3 LCPUFA, eicosapentaenoic acid (EPA) and docosahexaenoic acid (DHA) are associated with cardiovascular health, and they can be ingested in the form of fish or fish oil, or can be synthesized from the 18-carbon n-3 PUFA, $\alpha$ linolenic acid (ALA). ${ }^{1,2} \Delta 6$-Desaturase deficiency was diagnosed in a patient with clinical symptoms, suggestive of essential fatty acid deficiency. This was based on (a) deficient conversion of the n-6 $\Delta 6$-desaturase substrate, linoleic acid (LA), but not its product, $\gamma$ linolenic acid, to arachidonic acid (AA) and (b) lack of detection of mRNA on northern blot for the fatty acid desaturase 2 (FADS2) gene, which codes for $\Delta 6$-desaturase. ${ }^{3}$ The conclusion of $\Delta 6$-desaturase deficiency was supported by improvement in the patient's clinical condition after supplementation with AA. ${ }^{3}$ Further studies using this patient's fibroblasts suggested that the severely deficient expression of FADS2 was due to a thymidine nucleotide $(\mathrm{T})$ insertion between positions -941 and -942 upstream from the FADS2-coding region. ${ }^{4}$ The presence of a $\mathrm{T}$ in this position decreased binding of the transcription factor C/EBP and greatly decreased promoter activity in a promoter-reporter assay when compared with a $\mathrm{T}$ deletion at this position. ${ }^{4}$ Although the authors referred to this as an insertion mutation' for this patient, later population studies have revealed this to be a common polymorphism.

The polymorphism rs3834458 in the FADS2 promoter region involves $T /-$ alleles between positions -941 and -942 upstream from the FADS2 initiation codon. The frequency of the $\mathrm{T}$ allele in a Costa Rican cohort was $52 \%$ and clearly not a rare event. ${ }^{5}$ This cohort had higher plasma and adipose tissue EPA and AA levels in the T/T and $T /-$ genotypes compared with the $-/-$ genotype, suggesting that if this site did affect FADS2 activity, then the $\mathrm{T}$ insertion increased activity. ${ }^{5}$ To explore this discrepancy, we genotyped rheumatoid arthritis (RA) patients for the rs3834458 polymorphism to examine associations with fatty acid levels, and we examined the effect of the polymorphism on FADS2 promoter activity.

\section{MATERIALS AND METHODS}

Study population

Ninety-six RA patients who had low (average $<1 /$ week) or nil fish consumption and nil fish oil supplementation were selected from the Early Arthritis Clinic at the Royal Adelaide Hospital. The study was approved by the Royal Adelaide Hospital Research Ethics Committee.

\section{Genotyping}

The FADS2 rs3834458 (T/-) indel was genotyped by PCR-restriction fragment length polymorphism. Briefly, a 469-bp fragment of the FADS2 promoter region was amplified using the forward primer 5'-GGCAGTCTTTATTTGCTGGAGT-3' and reverse primer $5^{\prime}$-ACCTGATGCCGACACAAAG- $3^{\prime}$. The PCR product was digested with $2 \mathrm{U}$ of DdeI (New England Biolabs Inc., Arundel, Queensland, Australia) at $37^{\circ} \mathrm{C}$ for $3 \mathrm{~h}$. The digestion products were separated on $2 \%$ agarose gels, stained with ethidium bromide and visualized under UV light. There were two conserved restriction sites for $D d e$ in the amplified product, and a third site when the rs3834458 Tallele was present, resulting in 9, 76, 127 and 257-bp bands. In contrast, the deletion allele was characterized by 9,76 and 384-bp bands.

Fatty acid analysis

Plasma phospholipids were extracted and analyzed by gas chromatography, as previously described. ${ }^{6}$

\footnotetext{
${ }^{1}$ Rheumatology Unit, Royal Adelaide Hospital, Adelaide, South Australia, Australia; ${ }^{2}$ Basil Hetzel Institute for Medical Research, Queen Elizabeth Hospital, Adelaide, South Australia, Australia; ${ }^{3}$ Food Science, School of Agriculture, Food and Wine, University of Adelaide, Adelaide, South Australia, Australia

${ }^{*}$ Correspondence: Dr MK Gregory, Rheumatology Unit, Royal Adelaide Hospital, North Terrace, Adelaide, South Australia 5000, Australia. Tel: +61 882225581 ; Fax: +61 88222 5895; E-mail: melissa.gregory@health.sa.gov.au

Received 26 November 2010; revised 25 March 2011; accepted 3 May 2011; published online 1 June 2011
} 


\section{Plasmid constructs}

RNA was extracted from human leucocytes with the RNeasy kit (Qiagen, Doncaster, Victoria, Australia). PCR amplification of $\sim 1 \mathrm{~kb}$ of the FADS2 promoter (position -1119 to -114 relative to the FADS2 translation start codon) was performed using the forward primer $5^{\prime}$-GCGGTACCCCTGCCC AATGATGTGTCTGC- $3^{\prime}$ and reverse primer $5^{\prime}$-GGAAGCTTTTGTCTTCTCCT ACTCCTCCCACC- $3^{\prime}$. The PCR fragment was directionally cloned into the pGL3-Basic vector (Promega, Madison, WI, USA) using KpnI and HindIII restriction enzymes (Promega) to obtain the construct pGL3-F2P(T/T). Subsequently, a construct with the T deletion, pGL3-F2P $(-/-)$, was obtained using site-directed mutagenesis with the forward primer 5 -CCGCAATTCTTTTCAA GATTGTCTGACTAGAGGG- $3^{\prime}$ and reverse primer $5^{\prime}$-CCCTCTAGTCAGA CAATCTTGAAAAGAATTGCGG-3' ${ }^{\prime}$. Constructs were verified by sequencing.

\section{Cell culture and luciferase reporter assays}

The human hepatocellular liver carcinoma (HepG2) and mouse embryonic fibroblast (NIH/3T3) cells were seeded at a density of $1 \times 10^{5}$ cells per well in 24well plates (Nunc, Roskilde, Denmark). They were grown in Dulbecco's modified eagle's medium containing $10 \%$ fetal bovine serum, $20 \mathrm{~mm}$ HEPES and $2 \mathrm{mM} \mathrm{L}-$ glutamine at $37^{\circ} \mathrm{C}$ in a $5 \% \mathrm{CO}_{2}$ atmosphere for $24 \mathrm{~h}$. Each promoter-reporter construct $(0.5 \mu \mathrm{g})$ and internal control plasmid pRL-TK $(0.1 \mu \mathrm{g})$ was then transfected using Lipofectamine 2000 (Invitrogen, Mount Waverley, Victoria, Australia) according to the manufacturer's instructions. The pRL-TK plasmid was used as a transfection efficiency control. The Lipofectamine 2000/plasmid DNA complexes were incubated with the cells for $5 \mathrm{~h}$. The transfection medium was then replaced with fresh medium and the cells incubated for a further $43 \mathrm{~h}$. The pGL3-Basic vector (Promega) $(0.5 \mu \mathrm{g})$ was transfected with pRL-TK $(0.1 \mu \mathrm{g})$ to measure background luciferase activity. The reporter gene activity was measured using the Dual-Luciferase Reporter Assay System (Promega) in a GloMax-Multi Detection System (Promega). Transfection efficiency variations were normalized by dividing the promoter construct luciferase activity by the pRL-TK luciferase activity. The promoter activity was normalized relative to the activity of pGL3-Basic and expressed as relative luciferase units. Data represent three experiments, each performed in triplicate.

\section{Statistical analysis}

One-way analysis of variance with Tukey's post hoc test was performed using Graphpad Prism version 5.03. for Windows (Graphpad Software, San Diego, CA, USA). Statistical significance was set at $P<0.05$.

\section{RESULTS}

The genotype frequencies for the rs3834458 (T/-) indel were $-/-13$. $5 \%, T /-51 \%$ and $T / T$ 35.5\%, and were consistent with HardyWeinberg equilibrium. The frequency of the T-insertion allele was 0.61 . We evaluated the association of the FADS2 promoter polymorphism with plasma n-3 fatty acid indicators of FADS2 activity.
The ratios ALA/ALA+EPA and ALA/total n-3 represent substrate/ substrate+product(s). Higher ratios indicate lower FADS2 activity. The results from our subjects suggest FADS2 activity was highest with the $\mathrm{T}$ insertion, and the $\mathrm{T}$ allele was dominant in effect (Figure 1). FADS2 activity toward the ALA n-6 homolog, LA, was higher also in subjects with the $\mathrm{T}$ allele (data not shown).

To examine the functional effect of the polymorphism on FADS2 promoter activity, we transfected HepG2 and NIH/3T3 cells with the FADS2 promoter-reporter constructs containing the $\mathrm{T}$ insertion or deletion between -941 and -942 upstream from the start site. The luciferase reporter assays in both HepG2 and NIH/3T3 cells showed that the FADS2 promoter activity was not significantly affected by the T-insertion/deletion polymorphism (Figure 2).

\section{DISCUSSION}

The genotypes containing a $\mathrm{T}$ insertion between -941 and -942 from the FADS2 start site accounted for $86.5 \%$ of this population of Australian RA patients. The T-containing genotypes were present in Costa Rican and German populations at 52 and $89 \%$, respectively. ${ }^{5,7}$ This makes it unlikely that a severe fatty acid deficiency, later characterized as a $\Delta 6$-desaturase deficiency, was due to a $\mathrm{T}$ insertion at this site, as previously contended. ${ }^{4}$

The participants in our study were screened for low or nil consumption of fish and nil consumption of fish oil. Thus, it is probable that a large proportion of the EPA measured in the plasma

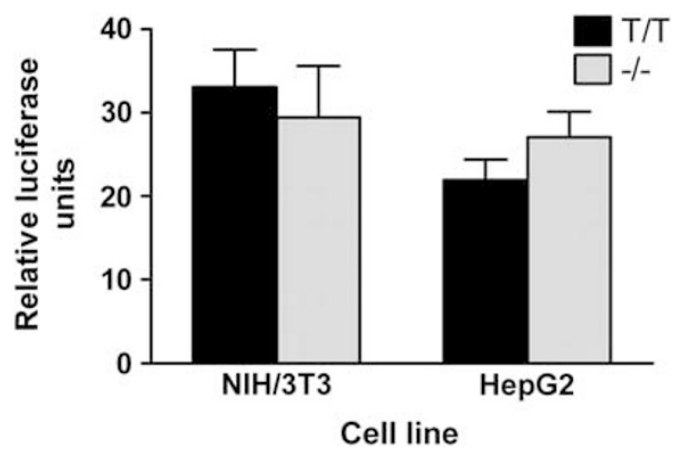

Figure 2 Relative functional activity of the FADS2 promoter-reporter constructs in NIH/3T3 and HepG2 cell lines. Data are the means \pm SD of three independent experiments performed in triplicate and normalized to pGL3-Basic. The activities of the promoter-reporter constructs were not statistically different in either cell line.

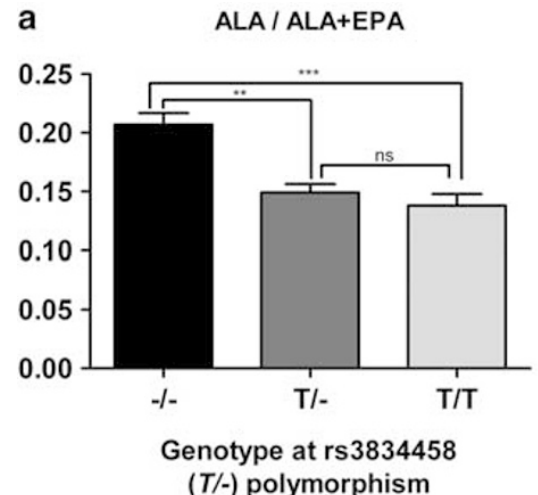

$(T /-)$ polymorphism b

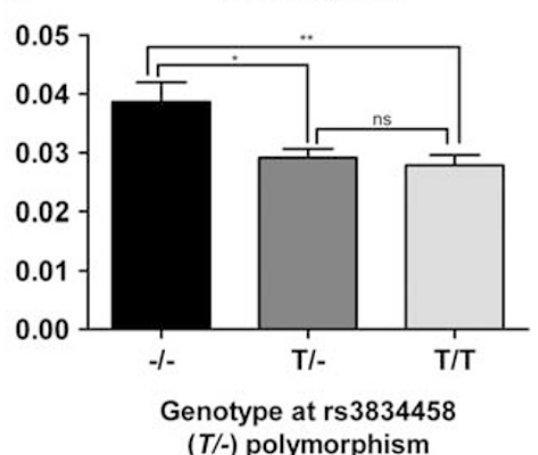

$(T /$-) polymorphism

Figure 1 Association of the polymorphism rs3834458 (T/-) in the FADS2 promoter region with fatty acid levels in plasma phospholipids of 96 RA patients. The ratios ALA/ALA+EPA (a) and ALA/Total n-3 (b) represent substrate/substrate+product(s). Total $n-3$ represents ALA+EPA+DPA+DHA. Data are the means \pm SD. ${ }^{*} P<0.05,{ }^{* *} P<0.01,{ }^{* * *} P<0.001$ and NS, not significant, $P>0.05$. 
was derived from metabolism of ALA initially by FADS2. Therefore, it is suggested that ALA/ALA+EPA is an indicator of FADS2 activity. The presence of the $\mathrm{T}$ allele was associated with higher FADS2 activity in the RA patients in this study. Adipose tissue EPA was higher in the presence of the $\mathrm{T}$ allele in the Costa Rican population, although without excluding fish or fish oil consumption the EPA value alone could not be used as a marker of FADS2 activity. ${ }^{5}$

Promoter-reporter constructs can be used to determine whether the alleles at this locus were functional as determinants of FADS2 promoter activity and consequent EPA levels. Conflicting results have been reported with the $\mathrm{T}$ insertion decreasing promoter activity by approximately sixfold when using the NIH/3T3 mouse fibroblast cell line, ${ }^{4}$ and having no effect on promoter activity when using the human hepatoma cell line HepG2. ${ }^{8}$ Therefore, we examined the effect of the $\mathrm{T}$ insertion at this site in both cell types. No effect of this polymorphism on FADS2 promoter activity was apparent.

Thus, although the indel polymorphism at -941 to -942 from the FADS2 translation start site is associated with fatty acid changes that reflect FADS2 activity, the polymorphism does not appear to directly affect promoter activity. However, the rs3834458 T allele is a tag for a common extended FADS2 haplotype identified in German Caucasians. $^{7}$ Therefore, enhanced FADS2 activity associated with the Tallele may be the product of other genetic variants in linkage disequilibrium with the $\mathrm{T}$ allele or even a haplospecific effect.

\section{CONFLICT OF INTEREST}

The authors declare no conflict of interest.

\section{ACKNOWLEDGEMENTS}

This work was supported by the National Health and Medical Research Council of Australia and the Australian Research Council.

1 Burdge GC, Calder PC: Conversion of alpha-linolenic acid to longer-chain polyunsaturated fatty acids in human adults. Reprod Nutr Dev 2005; 45: 581-597.

2 Kris-Etherton PM, Harris WS, Appel LJ: Fish consumption, fish oil, omega-3 fatty acids, and cardiovascular disease. Circulation 2002; 106: 2747-2757.

3 Williard DE, Nwankwo JO, Kaduce TL et al: Identification of a fatty acid delta6desaturase deficiency in human skin fibroblasts. J Lipid Res 2001; 42: 501-508.

4 Nwankwo JO, Spector AA, Domann FE: A nucleotide insertion in the transcriptional regulatory region of FADS2 gives rise to human fatty acid delta-6-desaturase deficiency. J Lipid Res 2003; 44: 2311-2319.

5 Baylin A, Ruiz-Narvaez E, Kraft P, Campos H: alpha-Linolenic acid, Delta6-desaturase gene polymorphism, and the risk of nonfatal myocardial infarction. Am J Clin Nutr 2007; 85: 554-560.

6 Metcalf RG, James MJ, Mantzioris E, Cleland LG: A practical approach to increasing intakes of $n-3$ polyunsaturated fatty acids: use of novel foods enriched with $n-3$ fats. Eur J Clin Nutr 2003; 57: 1605-1612.

7 Schaeffer L, Gohlke H, Muller M et al: Common genetic variants of the FADS1 FADS2 gene cluster and their reconstructed haplotypes are associated with the fatty acid composition in phospholipids. Hum Mol Genet 2006; 15: 1745-1756.

8 Lattka E, Eggers S, Moeller G et al: A common FADS2 promoter polymorphism increases promoter activity and facilitates binding of transcription factor ELK1. J Lipid Res 2010; 51: 182-191. 